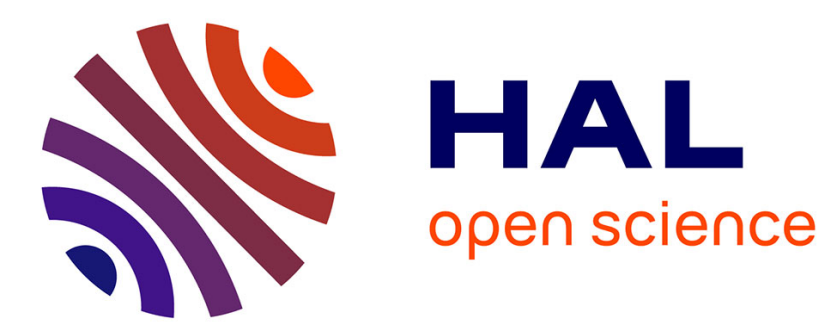

\title{
Light scattering cross section as a function of pair distribution density
}

Frédéric Gruy

\section{To cite this version:}

Frédéric Gruy. Light scattering cross section as a function of pair distribution density. Journal of Quantitative Spectroscopy and Radiative Transfer, 2009, 110 (3), pp.240-246. 10.1016/j.jqsrt.2008.10.003 . hal-00410253

\section{HAL Id: hal-00410253 \\ https://hal.science/hal-00410253}

Submitted on 19 Aug 2009

HAL is a multi-disciplinary open access archive for the deposit and dissemination of scientific research documents, whether they are published or not. The documents may come from teaching and research institutions in France or abroad, or from public or private research centers.
L'archive ouverte pluridisciplinaire HAL, est destinée au dépôt et à la diffusion de documents scientifiques de niveau recherche, publiés ou non, émanant des établissements d'enseignement et de recherche français ou étrangers, des laboratoires publics ou privés. 


\title{
Light-scattering cross section as a function of pair distribution density
}

\section{F. GRUY}

Département GENERIC (Géochimie, ENvironnement, Ecoulement, Réacteurs Industriels et Cristallisation), Centre SPIN - LPMG UMR CNRS 5148. Ecole Nationale Supérieure des Mines de Saint-Etienne. 158, Cours Fauriel. 42023 Saint-Etienne Cedex 2. gruy@emse.fr

keywords:

Mie; Light scattering; Cross section; Pair distribution

\begin{abstract}
This paper presents a simple method of approximation for calculating the scattering cross section for a random orientated convex particle illuminated by a non polarized electromagnetic wave. This method is proved efficient for sphere and spheroids as the scattering efficiency is smaller than one and as the material is optically either soft or hard.
\end{abstract}

\section{Introduction}

Since the nineteenth century, physicists have tried to calculate the optical properties of macroscopic bodies, particularly their scattering cross sections. This has led to several theories, known as approximations of the exact theory [1]:

$$
\begin{aligned}
& \text { * } d / \lambda<<1 \quad \text { : Rayleigh (R) approximation } \\
& \text { * } \quad d / \lambda<1 \text { et }|m-1|<<1 \quad \text { : Rayleigh-Debye-Gans (RDG) approximation } \\
& \text { * } \quad d / \lambda>1 \text { et }|m-1|<<1 \quad \text { : Anomalous Diffraction (AD) approximation } \\
& \text { * } d / \lambda>>1 \text { et } m-1>0 \text { : Fraunhofer Diffraction (FD) approximation }
\end{aligned}
$$

$\mathrm{d}$ and $\mathrm{m}$ are respectively the object size (for instance, the sphere diameter) and the ratio of the refractive indices for object and suspending medium.

The exact theory is due to Mie [2], who has solved the Maxwell's equations for the propagation of an electromagnetic wave around an object. Solution needs appropriate boundary conditions at the interface particle-medium. Since the Mie's ground-breaking paper, numerous works have been achieved in order to extend the method to bodies with more complex shape than the sphere one. Among these, one may emphasize investigations about the optical properties of sphere clusters. The latter require the use of sophisticated algorithms and the corresponding programming on computers. However, computations have been performed as the spheres, i.e the primary particles in the sphere cluster, are Rayleigh [3], Rayleigh-Debye-Gans [4] or Mie [5] scatterers.

As an obvious fact, the optical properties of a particle are related to its geometrical shape or its morphology. Several morphological functions have been proposed or appear in the calculations:

\footnotetext{
* the surface function for a convex particle, as dealt by Mie for a sphere

* the local pair (inter-particle distance) distribution density function for a fractal cluster consisting in RDG scatterers
} 
* the chord length distribution density for particle studied in the framework of $\mathrm{AD}$ approximation [6]

The different morphological functions are studied by means of integral geometry, which searches the relations between them [7]. The purpose of this paper is to search an approximate expression relating the light scattering cross section to a morphological function for a convex particle. Thus, one aims to calculate the light scattering cross section of simply shaped bodies by proposing a new approximation involving more recent results obtained for sphere clusters. The section 2 reviews the main results concerning the sphere clusters. The section 3 develops the new approximation. The section 4 shows the results of the comparison of the new approximation and exact theories when the body is a sphere or a spheroid.

\section{Scattering cross section for sphere clusters}

Let us consider a sphere cluster, consisting in $\mathrm{N}$ spherical primary particles with the same radius a. Its scattering cross section is denoted $\mathrm{C}_{\mathrm{N}}$. One will begin by treating the case of a cluster of RDG scatterers, itself being a RDG scatterer.

\section{Rayleigh-Debye-Gans approximation}

The volume element is the primary particle. Each volume element gives RDG scattering and does so independently of the other volume elements. The waves scattered in a given direction by all these elements interfere because of the different positions of the volume elements in space.

The scattering cross section (averaged over all the possible orientations) of the cluster obeys the relation

$$
C_{N}=N^{2} \int_{(4 \pi)} F_{1}(\vartheta) S(q) d \Omega
$$

with

$C_{1}=\int_{(4 \pi)} F_{1}(\vartheta) d \Omega$

$\vartheta, \Omega, q$ are respectively the scattering angle, the solid angle and the scattering vector $(q=2 k \sin (\vartheta / 2) ; k=2 \pi / \lambda)$. The function $F_{1}(\vartheta)$ corresponds to the primary particle. The structure factor $S(q)$ depends on the morphology of the cluster [4]:

$S(q)=\left[N+\sum_{j=l=1, j \neq l}^{N} \sin \left(q R_{j l}\right) /\left(q R_{j l}\right)\right] / N^{2}=\left[1+(N-1)\left\langle\sin \left(q R_{j l}\right) /\left(q R_{j l}\right)\right\rangle_{j l}\right] / N$

$R_{j l}$ is the distance between the centres of primary particles $\mathrm{j}$ and $\mathrm{l} .\langle\rangle_{j l}$ represents the average over all the pairs $(j, 1)$.

The structure factor is written within a continuous formulation:

$$
S(q)=\int_{R_{\min }}^{R_{\operatorname{mox}}} \sin (q R) /(q R) D_{P}(R) d R
$$

It appears in the expression of $S(q)$ the pair distribution density $D_{P}(R)$. Generally, R is the distance between two points inside the body. The distribution density function is normalized: 
$\int_{R_{\min }}^{R_{\max }} D_{P}(R) d R=1$

As far as a cluster of point scatterers is concerned:

$D_{P}(R)=\frac{1}{N(N-1)} \sum_{j, l} \delta\left(R-R_{j l}\right)$

$\delta$ is the Dirac function.

Then, the scattering cross section of the cluster can be written:

$C_{N}=N C_{1}\left[1+(N-1)\left\langle F^{R D G}\left(k R_{j l}\right)\right\rangle_{j l}\right] \simeq N^{2} C_{1}\left\langle F^{R D G}(k R)\right\rangle$

The mean value in equation 3 corresponds to:

$\langle X\rangle=\int_{R_{\min }}^{R_{\max }} X(R) D_{P}(R) d R$

where $\mathrm{X}$ is a given function of $\mathrm{R}$.

The function $F^{R D G}(k R)$ will not be expressed in this text, as it is not useful for the statement of the approximation.

Rayleigh Approximation

Generally, the object (primary particle, cluster ...) can be divided into smaller identical parts (elements, primary particle). Each element is polarisable. In the presence of a variable electric field, the element becomes an oscillating dipole, which itself creates an electromagnetic field. When an object is illuminated by an electromagnetic wave, each element receives the incident electric field and the one coming from the other elements. As a result, one may associate an oscillating dipole moment to each element. Thus, the object emits an electromagnetic wave (scattered wave), which includes the contribution of each oscillating dipole. Berry and Percival [3] calculated the scattering cross section of a cluster as the primary particle is a Rayleigh scatterer:

$$
C_{N}=6 \pi N|\rho|^{2} \frac{1+3(N-1) /(8 \pi)\left\langle\iint d \Omega e^{-i \overrightarrow{k_{s}} \cdot \overrightarrow{j_{j l}}} \cos \left(\overrightarrow{k_{i}} \cdot \overrightarrow{R_{j l}}\right)\left(1-\left(\overrightarrow{k_{s}} \cdot \overrightarrow{e_{i}} / k\right)^{2}\right)\right\rangle_{j l}}{k^{2}|1-1.5(N-1) \rho P|^{2}}
$$

with $\rho=\sin \delta e^{i \delta}$

$\delta=\frac{2}{3}(k a)^{3} \frac{m^{2}-1}{m^{2}+2}$

$P=\left\langle\frac{e^{i k R_{j l}}}{k R_{j l}} \cos \left(\overrightarrow{k_{i}} \cdot \overrightarrow{R_{j l}}\right)\left(1-\left(\vec{n} \cdot \overrightarrow{e_{i}}\right)^{2}+\left(3\left(\vec{n} \cdot \overrightarrow{e_{i}}\right)^{2}-1\right)\left(\frac{1}{\left(k R_{j l}\right)^{2}}-\frac{i}{k R_{j l}}\right)\right)\right\rangle_{j l}$

and $\vec{n}=\frac{\overrightarrow{R_{j l}}}{R_{j l}}$ 
$\overrightarrow{k_{i}}, \overrightarrow{k_{s}}, \overrightarrow{e_{i}}$ are respectively the incident wave number vector, scattered wave number vector and the incident polarization unit vector. Equation 4 also contains the pair distribution density for primary particles set. However, equation 4 includes a term (denominator) that is an estimate of the multiple scattering inside the cluster. The corresponding contribution is established from a mean field approximation (MFA). We take into account the multiple scattering because the condition $|m-1| \ll 1$ is not fulfilled. Nelson [8] performed a test of the MFA for the optics of fractal clusters. It was shown that multiple scattering should be considered and that MFA was suitable for aggregates consisting in several hundred soot particles. However, MFA was not tested for more general cases, as compact aggregates with a very large number of small primary particles.

Most often, the incident wave is randomly polarised and the object can randomly orientate. Thus, the optical properties are obtained after calculating an average over all the wave polarisation states and object orientations.

\section{Multi-sphere approximation for macroscopic body}

Let us consider a macroscopic body. It can be divided into identical parts small enough to behave as Rayleigh scatterers. Each scattering element will be considered as spherical. The scattering cross section of the body will be calculated in the framework of the Berry and Percival theory.

Several averages, translated as summation or integration, can be performed on equation 4 , as already done on expression 3:

$$
\begin{aligned}
& * \text { over solid angle (or scattering angle) } \\
& \text { * over the inter-particle (element) distances } R_{j l}:\langle\rangle_{j l} \\
& \text { * over the incident polarization direction (non polarized wave) }\langle\rangle_{\vec{e}_{1}} \\
& \text { * over all the space orientations of the cluster }\langle\rangle_{O}
\end{aligned}
$$

The first one is applied to the numerator of equation 4, the second one independently to numerator and denominator. By contrast, the two last ones should be applied to the whole expression. The application of average operators needs to get over mathematical difficulties. In order to simplify the mathematical treatment, one adopts the following approximation:

$$
\left\langle C_{N}\right\rangle_{\vec{e}_{1}, O}=\left\langle\frac{A}{|1+B P|^{2}}\right\rangle_{\vec{e}_{1}, O} \approx \frac{\langle A\rangle_{\vec{e}_{1}, O}}{\left|1+B\langle P\rangle_{\vec{e}_{1}, O}\right|^{2}}
$$

This leads to the expression (see also [4]):

$$
C_{N}=N C_{1} \frac{1+(N-1)\left\langle F_{i}^{R}\left(k R_{j l}\right)\right\rangle_{j l}}{\left|1-(N-1) \sin \delta e^{i \delta}\left(\left\langle F_{r}^{R}\left(k R_{j l}\right)\right\rangle_{j l}+i\left\langle F_{i}^{R}\left(k R_{j l}\right)\right\rangle_{j l}\right)\right|^{2}}
$$

with

$$
C_{1}=\frac{6 \pi \delta^{2}}{k^{2}}
$$




$$
\begin{aligned}
& F_{r}^{R}(x)=3\left[\cos (2 x)\left(2 x^{-1}-6 x^{-3}\right)+\sin (2 x)\left(1-5 x^{-2}+3 x^{-4}\right)\right] /\left(4 x^{2}\right) \\
& F_{i}^{R}(x)=3\left[\cos (2 x)\left(-1+5 x^{-2}-3 x^{-4}\right)+\sin (2 x)\left(2 x^{-1}-6 x^{-3}\right)+1+x^{-2}+3 x^{-4}\right] /\left(4 x^{2}\right)
\end{aligned}
$$

Moreover, let us consider the body consisting in a great number of Rayleigh scatterers, each one having a very small volume. The body volume is maintained constant:

$V=N a^{3}$ with $a \rightarrow 0$ and $N \rightarrow \infty$

Then,

$$
C_{\infty}=\frac{6 \pi}{k^{2}} \frac{\beta^{2}\left\langle F_{i}^{R}(k R)\right\rangle}{\left|1-\beta\left(\left\langle F_{r}^{R}(k R)\right\rangle+i\left\langle F_{i}^{R}(k R)\right\rangle\right)\right|^{2}}
$$

with

$$
\beta=\frac{V k^{3}}{2 \pi} \frac{m^{2}-1}{m^{2}+2}
$$

Equations 13-14 only contain $\mathrm{k}, \mathrm{m}$ and $\mathrm{V}$. The average in equation 13 includes the pair distribution density, that is a continuous function of the distance between two points of the body.

\section{Application to a sphere or a spheroid as a body}

\section{Sphere}

The pair distribution function for a sphere with radius b obeys the expression:

$$
b D_{p}(R)=D_{p, u}(u)=\frac{3}{16} u^{2}\left(u^{3}-12 u+16\right)
$$

with $u=R / b$ and $0<u<2$

One deduces from equation 13 the scattering efficiency factor for a sphere:

$$
Q_{\infty}=\frac{6}{\alpha^{2}} \frac{\beta^{2} G_{i}^{R}(\alpha)}{\left(1-\beta G_{r}^{R}(\alpha)\right)^{2}+\left(\beta G_{i}^{R}(\alpha)\right)^{2}}
$$

With $\alpha=k b, \beta=\frac{2}{3} \alpha^{3} \frac{m^{2}-1}{m^{2}+2}$

$\alpha$ is the size parameter and

$$
G_{X}^{R}(\alpha)=\int_{0}^{2 \alpha} F_{X}^{R}(v) D_{P, u}(v / \alpha) \alpha^{-1} d v
$$

With $X=i, r$

The $G_{X}^{R}(\alpha)$ functions can be analytically expressed.

Figure 1 shows $Q_{\infty}$ as a function of $\alpha$ for various $m$ values. 


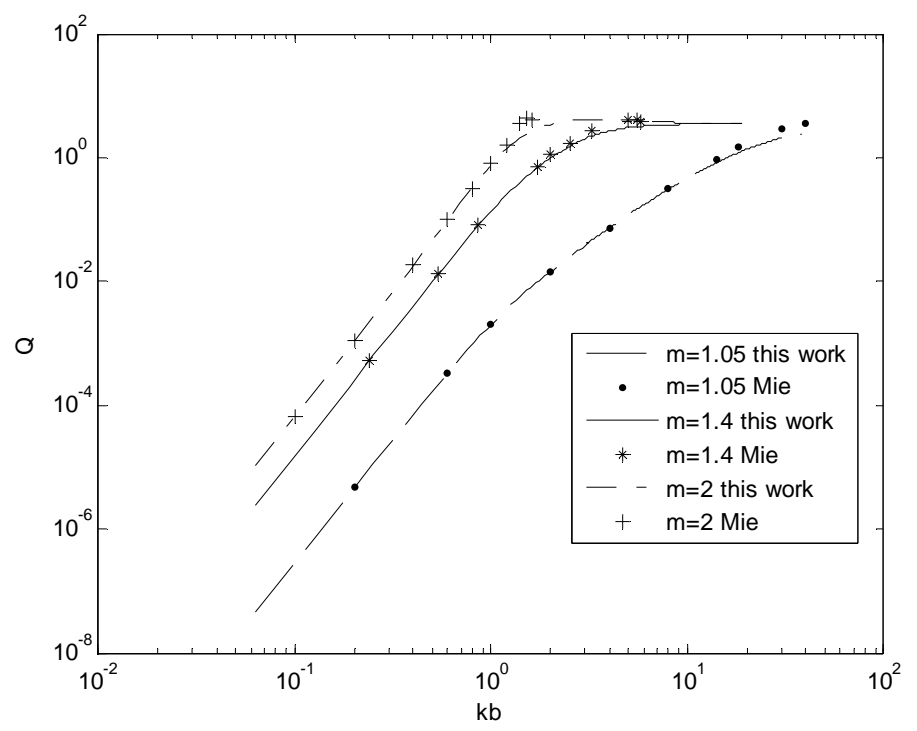

Fig 1a: $Q_{\infty}$ as a function of $\alpha$ for $\mathrm{m}=1.05,1.4$ and 2. logarithmic scale

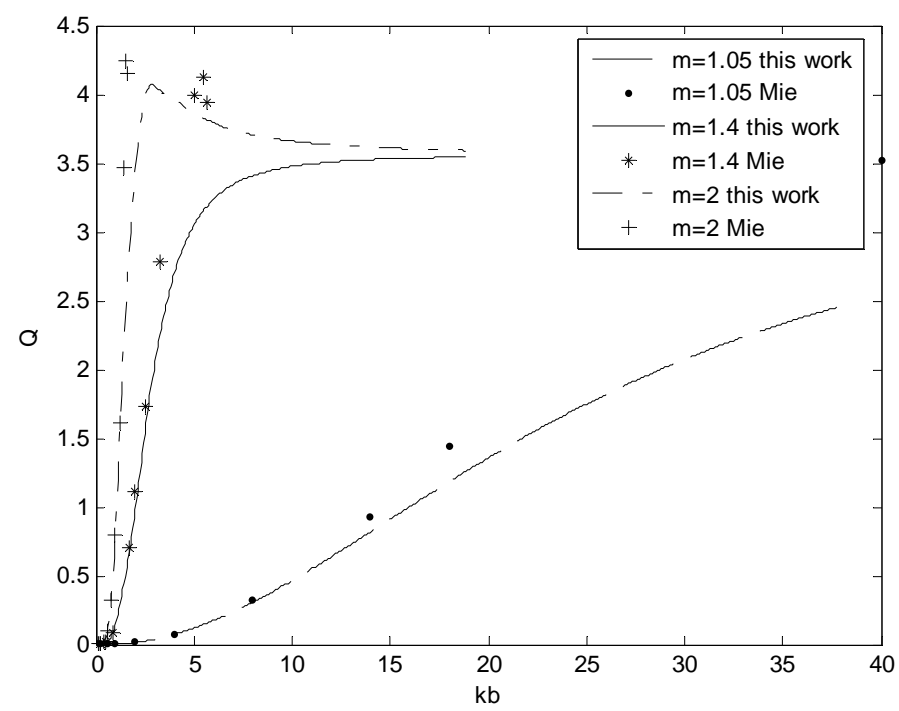

Fig $1 \mathrm{~b}: Q_{\infty}$ as a function of $\alpha$ for $\mathrm{m}=1.05,1.4$ and 2 . normal scale

One states that:

* $Q_{\infty}$ is a strongly increasing function of $\alpha$ for small $\alpha$ values. Then, depending on $\mathrm{m}$ value, $Q_{\infty}$ reaches a plateau $(\mathrm{m}<2)$ or a maximum followed by a plateau $(\mathrm{m}>2)$. The $Q_{\infty}$ value at the plateau is not depending on $m$ value: 3.58 . Hence, the global change of $Q_{\infty}$ with $\alpha$ is similar, but not exactly the same compared to the one deduced from Mie's theory. However, this is better than Rayleigh-Debye-Gans approximation for optically soft material at small and large size parameter.

* Deviation between equation 16 and exact theory is small as much as $Q_{\infty}<1$ for any m value. 


\section{Spheroid}

The pair distribution density for an oblate (semi-axis $b, \lambda b, \lambda b$ with $\lambda>1$ ) spheroid obeys the expression [9]:

$$
D_{p, u}(u)=12\left(\frac{u}{2 \lambda}\right)^{2}-9\left(\lambda+\frac{\operatorname{Ln}\left(\lambda+\left(\lambda^{2}-1\right)^{1 / 2}\right)}{\left(\lambda^{2}-1\right)^{1 / 2}}\right)\left(\frac{u}{2 \lambda}\right)^{3}+\frac{3}{4}\left(3 \lambda+2 \lambda^{3}+3 \frac{\operatorname{Ln}\left(\lambda+\left(\lambda^{2}-1\right)^{1 / 2}\right)}{\left(\lambda^{2}-1\right)^{1 / 2}}\right)\left(\frac{u}{2 \lambda}\right)^{5}
$$

with $u=R / b$ and $0<u<2$

$$
D_{p, u}(u)=\frac{9}{4} \frac{v}{\left(\lambda^{2}-1\right)^{1 / 2}}\left(\left(1-v^{2}\right)^{1 / 2}\left(2+v^{2}\right)+v^{2}\left(v^{2}-4\right) \operatorname{Ln}\left(\frac{1+\left(1-v^{2}\right)^{1 / 2}}{v}\right)\right)
$$

with $v=u / 2 \lambda$ and $2<u<2 \lambda$

Following the method of Garcia-Pelayo [9], the pair distribution density for a prolate (semiaxis $b, b, \lambda b$ with $\lambda>1$ ) spheroid obeys the expression:

$$
\begin{array}{ll}
D_{p, u}(u)=D_{p, u, 1}(u) & 0<u<2 \\
D_{p, u}(u)=D_{p, u, 1}(u)-D_{p, u, 2}(u) & 2<u<2 \lambda
\end{array}
$$

with

$$
\begin{aligned}
& D_{p, u, 1}(u)=\frac{3}{\lambda} u^{2}-\frac{9}{8} u^{3}\left(\frac{1}{\lambda^{2}}+\frac{A \tan \left(\lambda^{2}-1\right)^{1 / 2}}{\left(\lambda^{2}-1\right)^{1 / 2}}\right)+\frac{3}{64} u^{5}\left(\frac{1}{\lambda^{4}}+\frac{3}{2 \lambda^{2}}+\frac{3}{2} \frac{A \tan \left(\lambda^{2}-1\right)^{1 / 2}}{\left(\lambda^{2}-1\right)^{1 / 2}}\right) \\
& D_{p, u, 2}(u)=\frac{9 u}{4}\left(1+\frac{1}{8} u^{2}\right)\left(\frac{(u / 2)^{2}-1}{\lambda^{2}-1}\right)^{1 / 2}+\frac{9 u^{3}}{8}\left(\frac{1}{16} u^{2}-1\right) \frac{A \tan \left((u / 2)^{2}-1\right)^{1 / 2}}{\left(\lambda^{2}-1\right)^{1 / 2}}
\end{aligned}
$$

Then, the scattering cross section is deduced from equations 13-14. The result is presented as a scattering efficiency factor versus a surface-equivalent-sphere size parameter $\alpha_{s}$ following Mishchenko [10]. The scattering efficiency factor is defined as:

$Q_{\infty}=C_{\infty} /\left\langle S_{p}\right\rangle_{0}$

Where $\left\langle S_{p}\right\rangle_{o}$ is the average area of the particle geometric projection. Expressions for $\left\langle s_{p}\right\rangle_{o}$ can be found in [11].

As an illustration, figures $2 \mathrm{a}$ and $2 \mathrm{~b}$ present a comparison between the results of this work and numerical calculations obtained by the T-matrix method. The relative refractive index is fixed at $\mathrm{m}=1.311$. Results of the T-matrix method are presented as Figure 10.28 in [10]. 


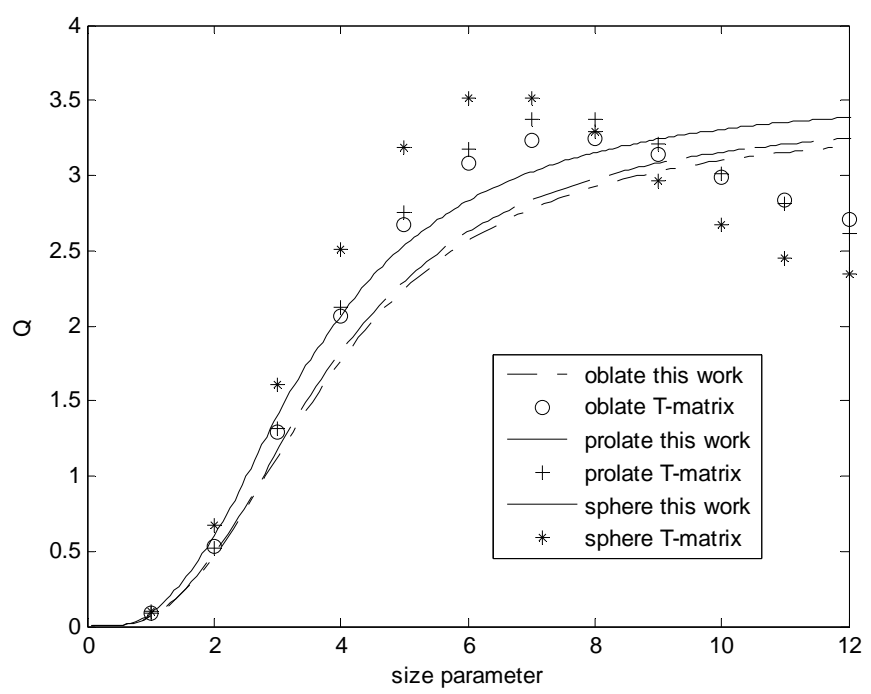

Fig 2a: $Q_{\infty}$ as a function of $\alpha_{s}$ for sphere and randomly oriented spheroids (oblate and prolate) with $\lambda=2(\mathrm{~m}=1.311)$.

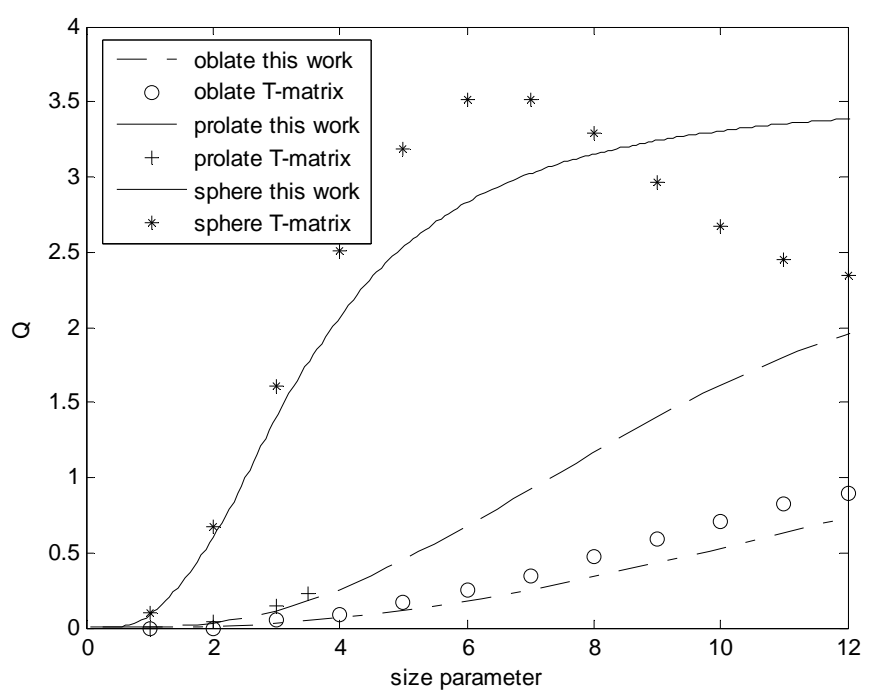

Fig $2 \mathrm{~b}: Q_{\infty}$ as a function of $\alpha_{s}$ for sphere and randomly oriented spheroids (oblate and prolate) with $\lambda=20(\mathrm{~m}=1.311)$.

Figures $2 \mathrm{a}$ and $2 \mathrm{~b}$ show that there is a small deviation ( $<15 \%$ for $\lambda=2$; $<30 \%$ for $\lambda=20$ in the case of $\alpha_{s}<12$ ) between scattering efficiency factor values coming from this work and these calculated with T-matrix method. The larger the size parameter is, the larger the deviation is. However, the main features of T-matrix results are retrieved: for instance, the curves for prolate spheroids are located above the curves for oblate spheroids. The deviation corresponding to spheroids behaves as the deviation for sphere. Thus, the relation $Q_{T-\text { matrix }}^{\text {sphere }} / Q_{\infty}^{\text {sphere }} \approx Q_{T-\text { matrix }}^{\text {spherd }} / Q_{\infty}^{\text {spheroid }}$ is found right within $5 \%$ for $\alpha_{s}<8$. 


\section{Conclusion}

Equation 13 enables an estimate of the mean scattering cross section for a random orientated body illuminated by a non polarized electromagnetic wave. It was proved efficient for a sphere as a body in the case of low and medium scattering efficiency and for any relative refractive index. This approximation was tried on other convex bodies as spheroids and was found suitable in this case. The shape of the body was taken into account by means of its pair distribution density, which is just a morphological function. For concave bodies, pair distribution density does not only characterize the content in material and consequently the body, but the mixture of material and medium of the convex shell. In this case, another density function would be probably more relevant and the previous approximation should be modified.

\section{References}

[1] Van de Hulst HC. Light scattering by small particles. New York: J . Wiley; 1957.

[2] Mie G. Beiträge zur Optik trüber Medien, speziell kolloidaler Metallösungen. Ann. Physik 1908;25:377-445.

[3] Berry MV, Percival IC. Optics of fractal clusters such as smoke. Optica Acta 1986; 33: 577-591.

[4] Khlebtsov NG. Spectroturbidimetry of fractal clusters: test of density correlation function cutoff. Appl Opt 1996; 35:4261-4270.

[5] Xu Y-l. Electromagnetic scattering by an aggregate of spheres. Appl Opt 1995; 34:45734588.

[6] Yang P., Zhang Z., Baum B.A., Huang H-L., Hu Y. A new look at anomalous diffraction theory (ADT): Algorithm in cumulative projected area distribution domain and modified ADT J ournal of quantitative spectroscopy \& radiative transfer 2004;89: 421-442.

[7] Santalo L, Integral geometry and geometric probability. Cambridge University Press, 2004.

[8] Nelson J. Test of a mean field theory for the optics of fractal clusters. J of Modern Optics 1989; 36: 1031-1057.

[9] Garcia-Pelayo R. Distribution of distance in a spheroid. J. Phys. A: Math. Gen. 2005; 38:3475-3482.

[10] Mishchenko MI, Travis LD, Lacis AA. Scattering, absorption and emission of light by small particles. Cambridge University Press; 2002.

[11] Min M, Hovenier JW, De Koter A. J QSRT 2003 ; 79-80 :939-951. 